\title{
Ligand Layer Engineering to Control Stability and Interfacial Properties of Nanoparticles
}

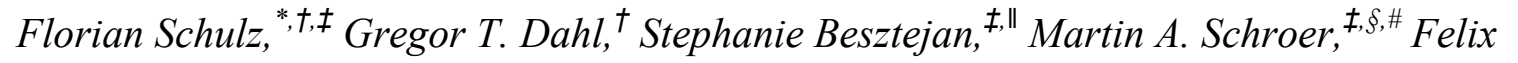 \\ Lehmkühler, ${ }^{\ddagger, \xi}$ Gerhard Grübel, ${ }^{\ddagger, \S}$ Tobias Vossmeyer, ${ }^{\dagger}$ Holger Lange ${ }^{\dagger, \ddagger}$
}

${ }^{\dagger}$ Institute for Physical Chemistry, University of Hamburg, Grindelallee 117, 20146 Hamburg, Germany.

* The Hamburg Centre for Ultrafast Imaging (CUI), Luruper Chaussee 149, 22761 Hamburg, Germany.

"Institute for Biochemistry and Molecular Biology, University of Hamburg, Martin-Luther-King Platz 6, 20146 Hamburg, Germany

$\S$ Deutsches Elektronen-Synchrotron DESY, Notkestr. 85, 22607 Hamburg, Germany

KEYWORDS. gold nanoparticles, poly(ethylene glycol), mixed ligand layers, nanomedicine, functionalization 


\section{ABSTRACT}

The use of mixed ligand layers including poly(ethylene glycol) based ligands for the functionalization of nanoparticles is a very popular strategy in the context of nanomedicine. However, it is challenging to control the composition of the ligand layer and maintain high colloidal and chemical stability of the conjugates. A high level of control and stability are crucial for reproducibility, upscaling and safe application. In this study, gold nanoparticles with well defined mixed ligand layers of $\alpha$-methoxypoly(ethylene glycol)- $\omega$-(11-mercaptoundecanoate) (PEGMUA) and 11-mercaptoundecanoic acid (MUA) were synthesized and characterized by ATR-FTIR-spectroscopy and gel electrophoresis. The colloidal and chemical stability of the conjugates was tested by dynamic light scattering (DLS), small-angle X-ray scattering (SAXS) and UV/Vis-spectroscopy based experiments and their interactions with cells were analyzed by elemental analysis. We demonstrate that the alkylene spacer in PEGMUA is the key feature for the controlled synthesis of mixed layer conjugates with very high colloidal and chemical stability and that a controlled synthesis is not possible using regular PEG ligands without the alkylene spacer. With the results of our stability tests, the molecular structure of the ligands can be clearly linked to the colloidal and chemical stabilization. We expect that the underlying design principle can be generalized to improve the level of control in nanoparticle surface chemistry.

\section{INTRODUCTION}

The ligand layer governs the interfacial properties of colloids and provides steric, and/or electrostatic stabilization. ${ }^{1-7}$ An effective stabilization is crucial for the reproducibility, processability, safety and thus for applications of colloidal nanomaterials, e.g. in nanomedicine, plasmonics, catalysis and energy conversion..$^{8-11}$ 
The stability of colloids encompasses their resistance to irreversible aggregation and/or disintegration of the ligand layer or the colloidal particle cores. Aggregation can be caused by high electrolyte concentrations in the case of electrostatically stabilized nanoparticles (NP), by mechanical compression (e.g. during centrifugation), or it can result from disintegration of the ligand layer by chemical decomposition, ligand stripping, or competitive displacement. ${ }^{7,12-14}$ Significant concentration losses can additionally result from irreversible adhesion of the NP to container or vessel walls. In complex biological media additional effects occur, the most prominent example being the so-called protein corona, i.e. additional layers of adsorbed proteins that affect e.g. the in vivo fate of the nanomaterial..$^{5,15-17}$ Thus, as a rough guideline, one can differentiate mechanical or physical stability, chemical stability and interactions of the ligand layer with its environment. Theoretical models of colloidal stability at the nanoscale extend the classical DLVO-theory to account for hydration forces, osmotic, entropic and enthalpic effects. ${ }^{718}$ Such effects, which are dictated by the ligand layer, cannot be neglected in the nanometer size-range.

To study the surface chemistry of colloids and the structure of ligand layers, gold nanoparticles (AuNP) serve as an ideal model system. Citrate stabilized AuNP are stable under ambient conditions and can be reacted with thiols for straightforward ligand exchange and functionalization. ${ }^{9,10}$ In the plethora of ligand layers on AuNP that have been explored, monolayers of poly(ethylene glycol)- (PEG-) based ligands and mixed ligand layers including such PEG-ligands play a prominent role. ${ }^{2,10,19-26}$ The so-called PEGylation allows for excellent stabilization of colloids and optimization of their interfacial properties, especially in the context of medical applications. Various studies explored the possibility of improving the performance of PEG-ligand layers by structural variations of the PEG-molecules. ${ }^{14,27-31}$ Alternative strategies 
have also emerged e.g. based on peptides or peptidols, ${ }^{19,32-34}$ and on zwitterionic ligands ${ }^{35,36}$ or zwitterionic ligand layers i.e. mixed layers from oppositely charged molecules. ${ }^{37,38}$

Because the use of mixed ligand layers is a promising strategy for improving or enabling various technological or medical applications of nanoparticles, ongoing research aims at a more detailed understanding of the involved surface chemistry. ${ }^{39-41}$ The same holds true for the surface chemistry of PEG-thiols on AuNP. ${ }^{13,14,42-44}$ Several studies have pointed out, that the grafting densities of typical PEG-thiol ligands on AuNP are significantly lower than those of small thiols. ${ }^{44-46}$ Thus, additional molecules can bind to vacant adsorption sites on the Au-surface, a process sometimes termed as backfilling. ${ }^{42-44}$ A low grafting density also facilitates competitive displacement of ligands. Such displacement of PEG ligands on AuNP has been shown for dithiols, such as dithiothreitol (DTT) $)^{13,14,47,48}$, but also for monothiols, e.g. different alkanethiols $^{42,49,50}$, and for cysteine at physiological concentrations. ${ }^{51}$ As a result the biorepulsive properties of the ligand layer and the provided stabilization are degraded. In recent studies, it has been shown that competitive displacement can be greatly reduced by using PEG-ligands with hydrophobic spacers connecting the thiol group with the PEG-moiety, e.g. $\alpha$ methoxypoly(ethylene glycol)- $\omega$-(11-mercaptoundecanoate) (PEGMUA). ${ }^{14,51}$ The use of hydrophobic spacers has been proven very effective for enhanced chemical stabilization and improved performance in biological media. In fact, for very small AuNP ( $\sim 2-8 \mathrm{~nm}$ in diameter), so-called monolayer protected cluster, this design principle is well established and often perused using commercially available (1-mercaptoundec-11-yl)tetra(ethylene glycol) (EG4MUA) or similar ligands $\left(\mathrm{EG}_{\mathrm{x}} \mathrm{MUA}\right)$, e.g. with six ethylene glycol units. ${ }^{411,52-54}$ However, for the stabilization and functionalization of larger AuNP (> $10 \mathrm{~nm})$ with PEG ligands this design principle is rarely taken into account. 
In this study, we explore the stability and properties of mixed ligand layers comprised of PEGMUA, a PEG-thiol with a hydrophobic spacer, and 11-mercaptoundecanoic acid (MUA). The main concept is that such mixed ligand layers enable the improved control of the ligand layer composition because the chemical structure of the adsorbates at the surface binding groups is similar. The concept is illustrated in Figure 1. Via the composition, properties like the surface charge of the AuNP-conjugates can thus be tuned precisely. As long as the mercaptoundecanespacer is used, well stabilized colloids can be synthesized, that resist multiple centrifugation steps at high forces $(20,000 \mathrm{~g})$ even at high MUA ratios, competitive ligand displacement and cyanide etching at high concentrations. We demonstrate that comparable stability and control of the ligand layer composition cannot be achieved with mixtures of standard methoxy PEG thiols (bearing no hydrophobic spacer) and MUA. Thus, the design principle of a hydrophobic spacer not only increases the stabilization of colloidal nanoparticles, but also enables a high level of control in the synthesis of AuNP with mixed self-assembled monolayers (SAMs) of PEG ligands and small thiols. 


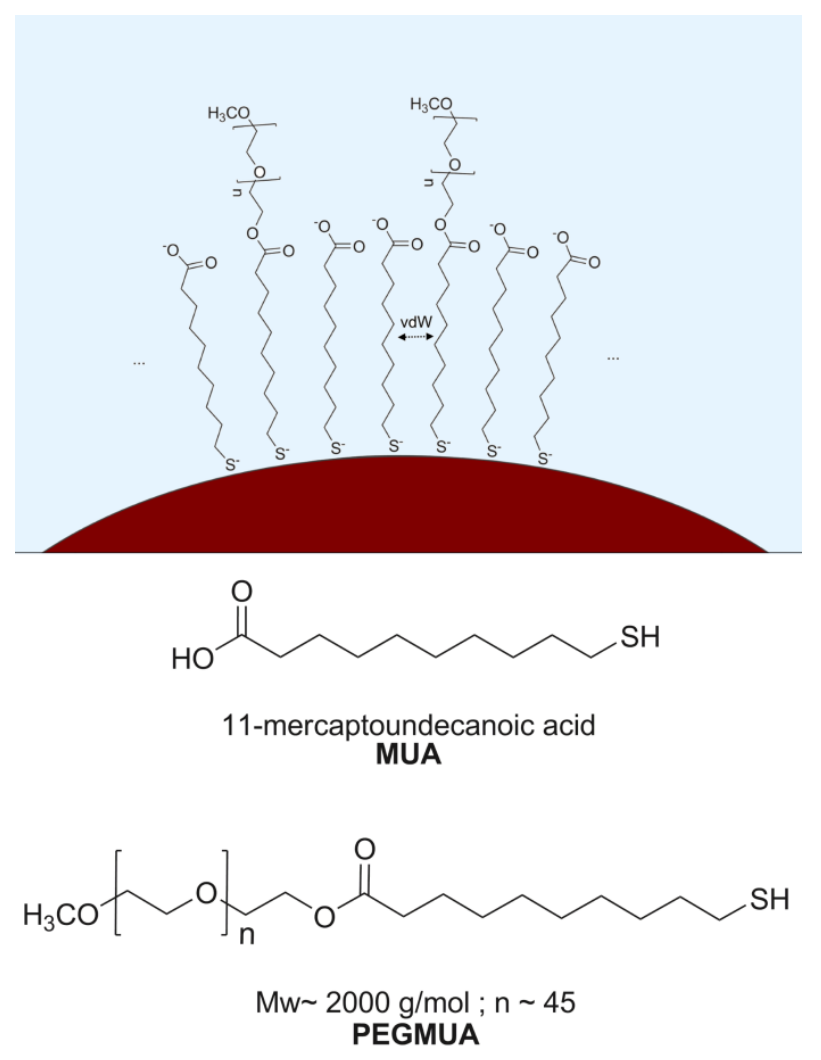

Figure 1. Illustration of a mixed ligand layer and structures of the according ligands. The structures of MUA and PEGMUA at the surface binding thiol group are the same and enable high grafting densities and stabilization of an inner hydrophobic layer by attractive van der Waals (vdW) interactions. The carboxylate groups of MUA affect the charge of the AuNP, depending on the $\mathrm{pH}$. The hydrophilic PEG moieties provide steric stabilization, solubility in water and improve the biocompatibility.

\section{EXPERIMENTAL SECTION}

\section{Materials}

Tetrachloroauric(III) acid ( $\geq 99.9 \%$ trace metal basis), trisodium citrate dihydrate ( $\geq 99.0 \%$ ), 11-mercaptoundecanoic acid (95\%), sodium chloride ( $\geq 99.0 \%)$ and dithiothreitol ( $\geq 98 \%)$ were ordered from Sigma-Aldrich, ethanol absolute (100.0\%) was obtained from VWR Chemicals. Ethylenediaminetetraacetic acid tetrasodium salt hydrate (EDTA) and citric acid monohydrate 
$(\geq 99.5 \%) \quad$ were obtained from Merck. $\alpha$-Methoxypoly(ethylene glycol)- $\omega-(11-$ mercaptoundecanoate) (PEGMUA, 2kDa or 5kDa) was synthesized as described previously. ${ }^{14} \alpha$ Methoxypoly(ethylene glycol)- $\omega$-(11-mercaptoundecanamide) (PEGMUA716, $M=716 \mathrm{~g} / \mathrm{mol}$, oligomer with 10 ethylene glycol units) was from Polypure (Polypure AS, Oslo, Norway). $\alpha$ Methoxy- $\omega$-mercaptopoly(ethylene glycol) (PEGSH, $2 \mathrm{kDa}$ ) was from Rapp Polymere (Tuebingen, Germany). Citrate-stabilized gold nanoparticles were synthesized and characterized as published earlier. ${ }^{55}$ Their TEM characterization is provided as Supporting Information, Figure S1. Fetal calf serum (FCS), Penicillin/Streptomycin and PBS were purchased from Lonza. Human serum was from Jackson ImmunoResearch (West Grove, PA, USA). Ham's F12 and RPMI1640 media were purchased from Life Technologies (now Thermo Fischer Scientific). Cell culture flasks and multiwell plates were purchased from Sarstedt (Nuembrecht, Germany). Ultrapure water (18.2 M $\Omega \mathrm{cm}$, Millipore) was used for all procedures.

\section{Functionalization of AuNP}

AuNP with mixed PEGMUA/MUA ligand layers. AuNP were functionalized by straightforward ligand displacement of citrate-stabilized AuNP using different mixtures of PEGMUA and MUA in an aqueous/ethanolic (1:1 vol.) solution. Eleven PEGMUA/MUA mixtures were prepared by mixing different volumes of an aqueous solution of PEGMUA $(1 \mathrm{mM})$ and an ethanolic solution of MUA (1 mM), yielding mixtures with the molar PEGMUA/MUA ratios of 100/0, 90/10, $80 / 20,70 / 30,60 / 40,50 / 50,40 / 60,30 / 70,20 / 80,10 / 90$ and 0/100. For each mixture, the solvent imbalance was compensated by addition of respective amounts of water and ethanol. The total concentration of thiol ligands was $0.5 \mathrm{mM}$ in $11.2 \mathrm{~mL}$ of water/ethanol (1:1 vol.) in all final mixtures. All ligand mixtures were stirred thoroughly for ca. $10 \mathrm{~min}$. To each mixture, $70 \mathrm{~mL}$ aqueous solutions of citrate-stabilized AuNP ( $c(\mathrm{AuNP}) \sim 5 \mathrm{nM}, c($ citrate $) \sim 2 \mathrm{mM}, \mathrm{pH} 5.5)$ were 
added at once and under constant stirring. After $10 \mathrm{~min}$, the AuNP solutions were subjected to centrifugation (20000 x g, $60 \mathrm{~min}$ ) and the supernatant was discarded, in order to remove free ligand molecules. Every sample was resuspended in $1.4 \mathrm{~mL}$ of water using a vortex mixer and further purified by 3 centrifugation steps ( $20000 \mathrm{x} \mathrm{g}, 15 \mathrm{~min}$ ), each with subsequent replacement of $1.4 \mathrm{~mL}$ of the supernatant by water and resuspension. The final AuNP concentrations of the samples were in the range 120-180 $\mathrm{nM}$. AuNP conjugates with other PEGMUA/MUA ratios (e.g. 25 and $75 \%$ ) were prepared accordingly. All samples were stored at $7{ }^{\circ} \mathrm{C}$ and no destabilization or indications of changed layer composition (e.g. due to hydrolysis of PEGMUA, tested by ATR-FTIR spectroscopy as described in the main text) were found after storage for $>$ 12 months. AuNP with mixed layers of PEGSH and MUA were prepared the same way, but aggregated during the centrifugation steps as described in the main text. AuNP coated with just PEGSH were stored under the same conditions as the AuNP@PEGMUA/MUA conjugates and were also stable for $>12$ months. AuNP concentrations were adjusted and determined by UV/Vis spectroscopy as described by Haiss et al. ${ }^{56}$

\section{Characterization}

ATR-FTIR spectroscopy. FTIR-spectra were recorded with a Varian 660 FTIR spectrophotometer equipped with a PIKE MIRacle ${ }^{\mathrm{TM}}$ ATR sampling accessory. Spectra of PEGMUA and MUA were recorded from the pure solids pressed onto the crystal (diamond) with a high pressure clamp. To record spectra of AuNP samples, 2-3 $\mu 1$ of the according concentrated solutions (c(AuNP) $\sim 1 \mu \mathrm{M})$ were pipetted directly onto the crystal. After evaporation of the solvent (water, usually within 15-30 min) spectra were recorded. Afterwards the crystal was thoroughly cleaned with water and ethanol. 32 scans in absorbance mode with $4 \mathrm{~cm}^{-1}$ resolution were recorded for each measurement. 
UV/Vis Spectroscopy. Absorbance measurements were carried out using a Perkin-Elmer Lambda 25 or a Varian Cary 50 spectrometer. UV microcuvettes sealed with lids (Plastibrand, Carl Roth GmbH, Karlsruhe, Germany) were used for all experiments.

Transmission Electron Microscopy (TEM). TEM measurements were performed using a Jeol JEM-1011 instrument operating at $100 \mathrm{kV}$. For TEM sample preparation, ten microliters of sample solution were drop-casted onto a carbon-coated copper grid, which was placed on a glass slide, and left to dry for at least $24 \mathrm{~h}$.

Gel electrophoresis. For gel electrophoresis analysis, a $0.5 \%$ agarose gel was prepared by first dissolving $25 \mathrm{~g}$ of agarose in $500 \mathrm{~mL} 1 \mathrm{X}$ TAE buffer solution (40 mM Tris, $40 \mathrm{mM}$ acetic acid, $1 \mathrm{mM}$ EDTA, in water). This was accelerated by heating the solution up to approximately $95^{\circ} \mathrm{C}$ in a microwave oven. It was cooled down for $5 \mathrm{~min}$ at room temperature and poured into the gel box, which was equipped with a comb parallel to the electrodes. After complete polymerization, the comb was removed carefully and the box was filled with $1 \mathrm{X}$ TAE buffer, covering the entire gel and electrodes. $15 \mu \mathrm{L}$ of each sample were mixed with $3 \mu \mathrm{L}$ gel loading dye (DNA Gel Loading Dye (6X) R0611, Thermo Scientific, USA) and transferred carefully into the respective pockets in the gel. The box was covered and between the electrodes a direct voltage of $120 \mathrm{~V}$ was applied. After 200 min, the voltage was turned off. Photographic images of the gel were taken after 5, 50 and 200 minutes.

Small-angle X-ray scattering (SAXS). SAXS measurements were performed at beamline I22, Diamond Light Source, Didcot, U.K. Two-dimensional scattering patterns were recorded with a Pilatus 2M detector at a sample-detector distance of $6.704 \mathrm{~m}$ using a $160 \times 300(\mathrm{v} \times \mathrm{h}) \mu \mathrm{m} \times$-ray beam with an $\mathrm{x}$-ray energy of $\mathrm{E}=18 \mathrm{keV}$ (wavelength $\lambda=0.68 \AA$ ). The exposure time per SAXS pattern was $1 \mathrm{~s}$. The SAXS patterns were azimuthally integrated and the background signals 
from the buffer that were measured separately were subtracted from these. For the diluted suspensions, the SAXS signal is directly proportional to the particle form factor $\mathrm{P}(\mathrm{Q})$. The form factor was modeled by that of polydisperse spheres. ${ }^{57}$ Due to the low scattering contrast of the PEGMUA shell in water $\Delta \rho=40 \mathrm{e}^{-} / \mathrm{nm}^{3}$ compared to that of the gold core $\left(\Delta \rho=4300 \mathrm{e}^{-} / \mathrm{nm}^{3}\right)$ the dominant scattering contribution arises from the latter one, i.e. the PEGMUA shell is not visible for the diluted suspensions.

\section{Stability Tests}

Stability against Cyanide Etching. For each etching experiment $800 \mu \mathrm{L}$ of the AuNP sample were transferred into a UV cuvette and mixed thoroughly with $200 \mu \mathrm{L}$ of an aqueous $\mathrm{KCN}$ solution ( $1 \mathrm{M}$ ), yielding a $\mathrm{KCN}$ concentration of $0.2 \mathrm{M}$ in the cuvette. The AuNP concentrations were 5-6 nM after mixing. The spectrometer was operated in cycle mode. Absorbance spectra in the range of $200-800 \mathrm{~nm}$ were recorded every $10 \mathrm{~min}$ for $18 \mathrm{~h}$, collecting a total of 108 spectra per sample. During the measurement, the cuvettes remained sealed with a lid and were not shaken or stirred. The control experiments with PEGSH coated AuNP, AuNP@PEGSH, were prepared accordingly, but the final $\mathrm{KCN}$ concentration was $0.1 \mathrm{M}$. All etching experiments were performed twice to test for reproducibility.

Stability against Competitive Adsorption of Dithiothreitol (DTT). $500 \mu \mathrm{L}$ of each AuNP sample were transferred into a UV cuvette and mixed thoroughly with an aqueous solution containing DTT $(2 \mathrm{M})$ and $\mathrm{NaCl}(0.8 \mathrm{M})$, yielding concentrations of DTT and $\mathrm{NaCl}$ in the mixture of $1 \mathrm{M}$ and $0.4 \mathrm{M}$, respectively. The AuNP concentrations were 5-6 $\mathrm{nM}$ after mixing. Absorbance spectra in the range of $200-800 \mathrm{~nm}$ were recorded in cycle mode every $2 \mathrm{~min}$. For each experiment, 240 spectra were recorded over a period of $8 \mathrm{~h}$, during which the cuvette was neither shaken nor stirred. 


\section{Internalization Assay}

PC3 cells were cultivated in a 1:1 mixture of Ham's F12 and RPMI 1640, supplemented with $10 \%$ fetal calf serum (FCS) and $1 \%$ Penicillin/Streptomycin. LNCaP cells were cultivated in RPMI 1640 mixed with $20 \%$ fetal calf serum and $1 \%$ Penicillin/Streptavidin. For the experiments, PC3 cells were seeded with a cell density of 37,500 cells per well in a 24 -well plate, while LNCaP cells were seeded with a density of 50,000 cells per well 24 hours before the experiments. At the day of the experiments, the confluency was checked via light microscopy yielding approximately $70 \%$ confluency for both cell lines. The cells were washed two times with PBS, new media added, and the AuNP solutions were added to the cells to the desired final AuNP concentration. The cells were incubated with the AuNP for 1 and $24 \mathrm{~h}$. After the incubation, the cells were washed twice so that there was no red color of the AuNP visible in the supernatant. Further, the cells were lysed with lysis-buffer $(0.1 \mathrm{M}$ Tris-HCl pH10, 0.1\% TritonX100) and prepared for element analysis. $200 \mu 1$ of each sample were reacted with $200 \mu 1$ freshly prepared aqua regia overnight, then filled up to $1000 \mu \mathrm{l}$ and analyzed by GF-AAS to quantify the gold content.

Graphit furnace atom absorption spectrometry (GF-AAS). GF-AAS measurements were performed with a ContrAA-700 AAS-spectrometer (Analytik Jena, Germany) at 242,795 nm. The limit of detection (LOD) was $10 \mu \mathrm{g} / \mathrm{l}$. Measurements were performed in triplicates and the relative standard deviation of the mean was $1-5 \%$.

\section{Toxicity test lactate dehydrogenase (LDH) activity assay}

To determine the toxicity of the AuNP, 7500 PC3 cells were seeded per well in a 96-well plate 24 hours before the test. AuNP conjugates were added to the PC3 cells (final $c(\mathrm{AuNP})=20 \mathrm{nM}$ ) and incubated for one and $24 \mathrm{~h}$. Each sample was added to three different wells. The 
supernatants were collected after the different incubation times and stored at $-20^{\circ}$ until the measurement. For the LDH-assay the commercially available LDH-Cytotoxicity Colorimetric Assay Kit II (Biovision Inc., USA) was used and the samples and cells prepared following the manufacturer's protocol. The analysis of the samples was performed photometrically at $450 \mathrm{~nm}$ using a plate reader (Tecan, Switzerland).

\section{RESULTS}

\section{Synthesis and characterization of mixed ligand layers}

A protocol for the highly reproducible synthesis of citrate-stabilized gold nanoparticles (AuNP) with very low dispersity (5-8 \%) and high uniformity was published recently. ${ }^{55}$ Here, such AuNP were functionalized with mixtures of PEGMUA and MUA by simply mixing the reagents at room temperature (see the Experimental Section). The TEM characterization of AuNP used herein is provided as Supporting Information, Figure S1.

The footprint of different thiols or, more general, different adsorbates, can differ strongly. While for MUA and other small alkyl thiols a footprint of $0.17 \mathrm{~nm}^{2}$ is established, ${ }^{3,58}$ PEG molecules, thiolated DNA, peptides and proteins can have much larger footprints up to several $\mathrm{nm}^{2}$ per molecule. Therefore, the assessment of the ligand layer composition based on the composition of the reaction mixture of ligands before conjugation is difficult: ${ }^{59}$ Will the ratio of the ligand reaction mixture transfer to the number ratio of ligands bound to the particle surface or the relative areas they occupy? Or will one ligand bind preferentially? Size, polarity, solubility and steric demand of the ligands additionally affect the kinetics and enthalpy of their adsorption and therefore the composition of the mixed ligand layer. ${ }^{39,40,60,61}$ By using the same spacer for the adsorbed ligands, some of these complications are avoided. The footprint is more homogeneous 
and the chemical structure near the thiol group is the same. This results in better control of the ligand layer's composition that is closer to the composition of the reaction mixture than for ligands with completely different footprints and affinities. It is known that for solutions containing two thiols, adsorption of the thiol with the longer alkyl chain is preferred, ${ }^{60}$ a binary SAM is more homogeneous when the thiols have the same number of methylene units ${ }^{61}$ and that attractive van der Waals (vdW) interactions add significantly to the adsorption enthalpy. ${ }^{62}$ Therefore, a mixture of thiols with long alkyl chains, all of the same length, should be a good choice for the formation of stable and well-defined mixed ligand layers on AuNP. In the following sections we will show that this is indeed the case for mixtures of MUA and PEGMUA ( $2 \mathrm{kDa}$ ), even though their molecular masses differ by a factor of $\sim 10$. We note that for AuNP with diameters $>4.4 \mathrm{~nm}$ the structure of alkanethiolate SAMs on the gold surface has been shown to be similar to SAMs on planar gold surfaces, i.e. curvature effects are not expected to play a dominant role for the AuNP used in this study. ${ }^{63}$

To directly analyze the mixed ligand layer compositions we used Fourier transform infrared spectroscopy with attenuated total reflection (ATR-FTIR). Distinct modes of MUA and PEGMUA can be identified and utilized to quantify the relative ratios of the according ligands in the mixed ligand layer as shown in Figure 2. The data show a clear trend evidencing a decreasing PEG ratio in the ligand layer of the synthesized and purified conjugates with increasing MUA ratio used in the synthesis. This indicates that the composition of the ligand layer can be controlled by the experimental conditions. 


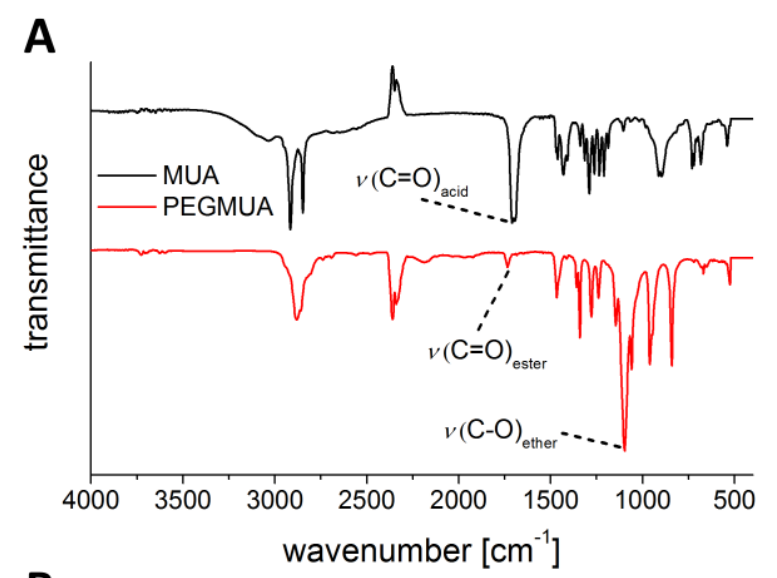

B

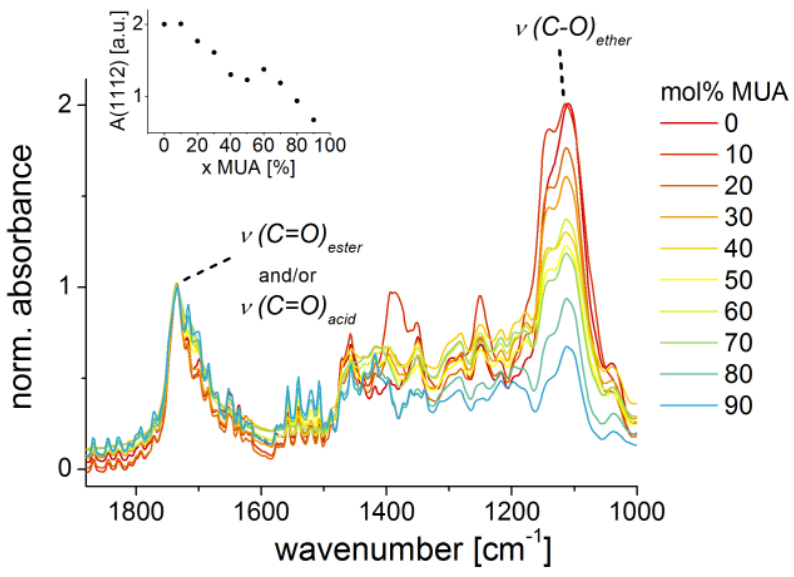

Figure 2. ATR-FTIR spectra of ligands MUA and PEGMUA (A) and AuNP conjugates with mixed ligand layers (B). A distinct spectral feature of MUA is the strong acidic $\mathrm{C}=\mathrm{O}$ vibration at $1712 \mathrm{~cm}^{-1}$. The close ester $\mathrm{C}=\mathrm{O}$ vibration of PEGMUA at $1730 \mathrm{~cm}^{-1}$ is much weaker. For PEGMUA, the ether C-O vibrations at $\sim 1100 \mathrm{~cm}^{-1}$ are characteristic (A). In the spectra of AuNP conjugates with different mixed ligand layers the backgrounds were accounted for with a second order polynomial and subtracted. The spectra were then normalized at $1720 \mathrm{~cm}^{-1}$ to analyze the changing intensity ratio of the bands at $1720 \mathrm{~cm}^{-1}$ and at $\sim 1100 \mathrm{~cm}^{-1}$ (B). The band at $1720 \mathrm{~cm}^{-1}$ is dominated by the acidic $\mathrm{C}=\mathrm{O}$ vibration of MUA. The band at $\sim 1100 \mathrm{~cm}^{-1}$, related to PEGMUA, decreases in intensity with increasing MUA ratio (assumed MUA mole percent based on the synthesis are indicated by the color code). The correlation of the relative intensity at 1112 $\mathrm{cm}^{-1}$ (maximum of the band at $\sim 1100 \mathrm{~cm}^{-1}$ ) and the MUA mole percent of the MUA/PEGMUA 
reaction mixture is plotted in the inset. Figure part A is adapted with permission from ref. 64 Published by The Royal Society of Chemistry.

The control of the conjugates ${ }^{6}$ charge via ligand layer composition is demonstrated by gelelectrophoresis experiments. The migration of the conjugates in an externally applied electric field is in complete accordance with their assumed ligand layer composition and surface charge (Figure 3), underlining the level of control in the synthesis. The AuNP with just MUA migrated much faster than all other conjugates, indicating a strong attenuation of electrophoretic mobility by the PEG moiety resulting from an increased hydrodynamic diameter. The electrophoretic mobility is affected by both, the hydrodynamic diameter and the charge of the AuNP and the effects cannot be separated. Considering that the change of AuNPs' hydrodynamic diameter e.g. from 0 to $10 \%$ MUA content should be very small but their migration differs significantly (compare Figure 3, bottom) we assume that the effect of charge is dominating in this experiment. 


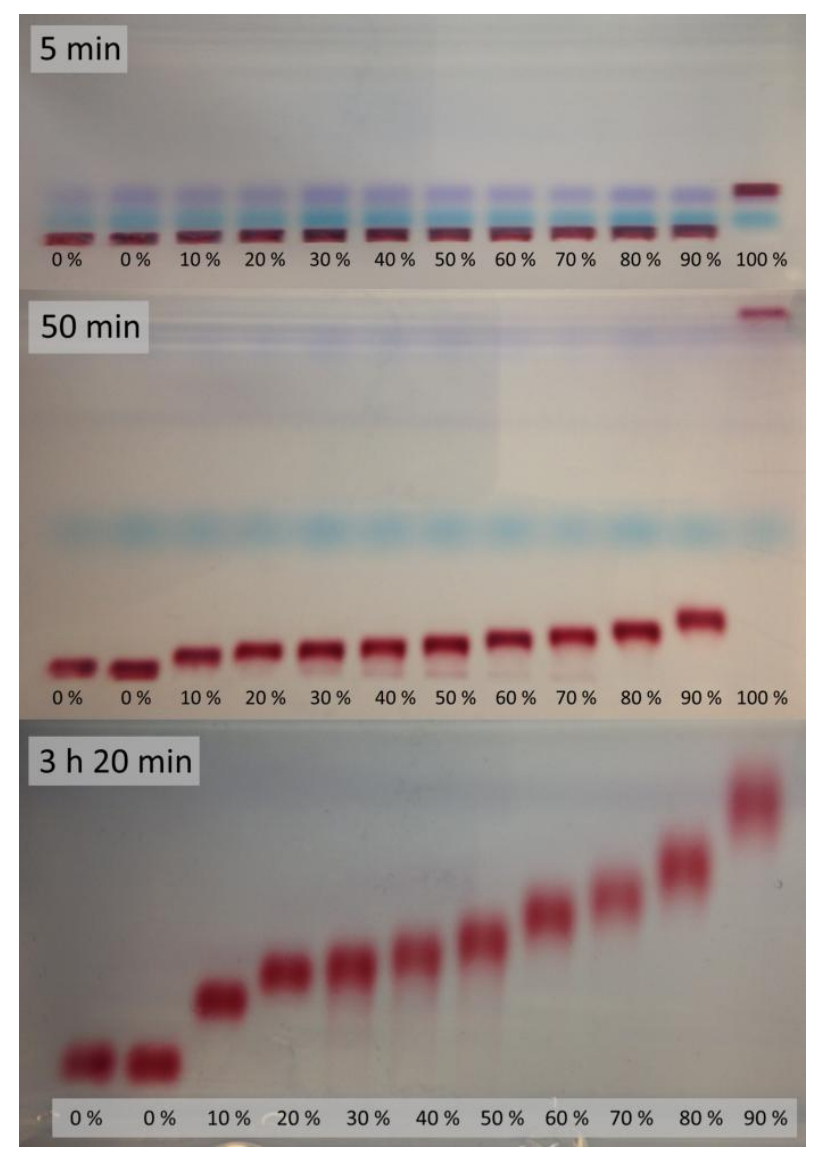

Figure 3. Migration of AuNP with different mixed ligand layers in an externally applied field $(120 \mathrm{~V})$ in a $0.5 \%(\mathrm{w} / \mathrm{v})$ agarose gel. Blue and violet bands originate from the loading dyes bromophenol blue and xylene cyanol. The mole percent of the MUA/PEGMUA reaction mixture is indicated at each band. The AuNP with only MUA (100\%) migrated much faster than all other conjugates and are not shown in the photograph after $3 \mathrm{~h} 20 \mathrm{~min}$.

The surface charge of colloids affects many of their properties and interactions. For example the self-assembly of the AuNP conjugates was found to depend strongly on the presence of MUA in the ligand layer (Figure 4). Thus, precise control of the surface charge provides a tool not only for fundamental studies but possibly also for manipulating the assembly of nanoparticles. 


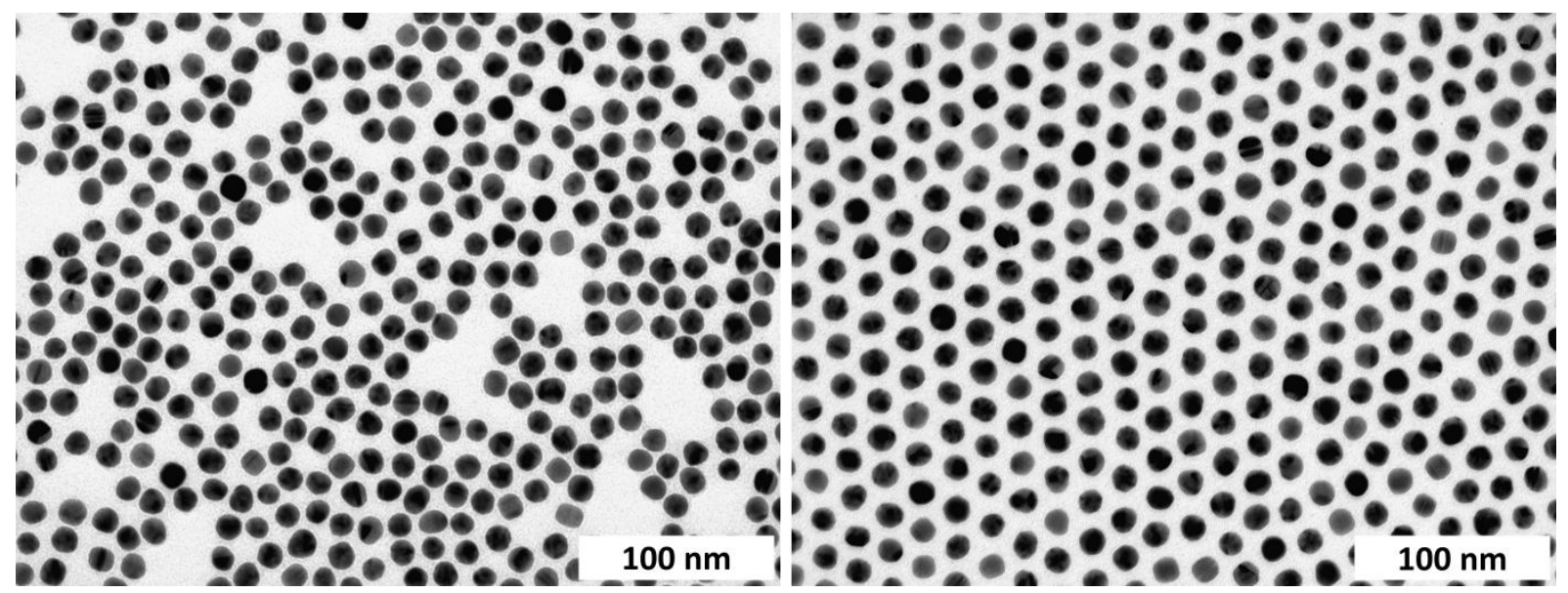

Figure 4. Transmission electron microscopy (TEM) images of AuNP conjugates with $75 \%$ (left) and $0 \%$ MUA (right). Purified aqueous solutions of the according AuNP conjugates were dropcasted onto carbon coated copper grids and analyzed by TEM after drying at room temperature overnight. The self-assembly of the conjugates is strongly affected by their ligand layers' composition.

In summary, the synthesis of mixed ligand layers of PEGMUA and MUA is straightforward and allows control of the AuNPs' surface charge. The layer composition affects the self assembly of the AuNP and can more generally be expected to affect the interaction of the AuNP with different substrates, an aspect interesting in the context of nanofabrication.

\section{Colloidal stability}

PEGMUA with a molecular weight of $2 \mathrm{kDa}$ (or $5 \mathrm{kDa}$ ) provides AuNP with high stabilization against centrifugation. When aqueous solutions of AuNP@PEGMUA2k (or 5k) are centrifuged at 20,000 x g for 20 minutes no signs of aggregation can be found. Thus, these conjugates can be purified and concentrated with negligible losses. This mechanical stability allows to achieve very high particle concentrations by centrifugation.

When comparing AuNP conjugates with mixed layers of PEGMUA und MUA, high stability against irreversible aggregation induced by centrifugal forces was observed for MUA ratios up to 
$90 \%$. Significant aggregation was only observed for $100 \%$ MUA conjugates. Slight aggregation was observed for some samples with MUA-ratios $>75 \%$. Formation of loose aggregates during centrifugation could be reversed by ultrasonication. At very high AuNP concentrations ( $>1 \mu \mathrm{M})$, however, the pellets of mixed layer conjugates are increasingly difficult to redisperse in comparison to the pure AuNP@PEGMUA conjugates. This underlines that the stability against centrifugation does not only depend on the parameters of centrifugation and the particle size, but also on their concentration. At higher particle concentrations the compression of lower parts of the pellet becomes more severe due to its increasing mass.

In order to evaluate the colloidal integrity of concentrated AuNP solutions, small-angle X-ray scattering (SAXS) measurements were performed (Figure 5). The optical density of such concentrated samples is far too high for UV/Vis spectroscopy and SAXS provides a complementary technique to obtain structural information, in particular to determine the presence of aggregates in solution. At $c($ AuNP $)=200 \mathrm{nM}$ no destabilization of conjugates with 25,50 or $75 \%$ MUA ratio was observed. The experimental SAXS curves for all samples exhibit the same shape, i.e. position of the pronounced minima as well as similar forward scattering, for the whole Q-range determined, indicating their integrity. In more detail, the radii and polydispersities obtained by fitting the SAXS data were in excellent agreement with TEM analysis (Table S1) and no strong contributions at low Q-values were observed for all samples studied, indicating little or no aggregation. In conclusion, very high stability against irreversible aggregation can be obtained for mixed layer conjugates, even with MUA-ratios as high as $75 \%$. A difference in stabilization was observed only at very high particle concentrations ( $>4 \mu \mathrm{M}$ AuNP $=40 \mathrm{mg} / \mathrm{ml}$ $\mathrm{Au}$ ) in that conjugates with $100 \%$ PEGMUA exhibited immaculate colloidal integrity, whereas 
for conjugates with mixed PEGMUA/MUA layers indications of aggregates were observed (Figure S2). The colloidal integrity of the main population, however, was unaffected.
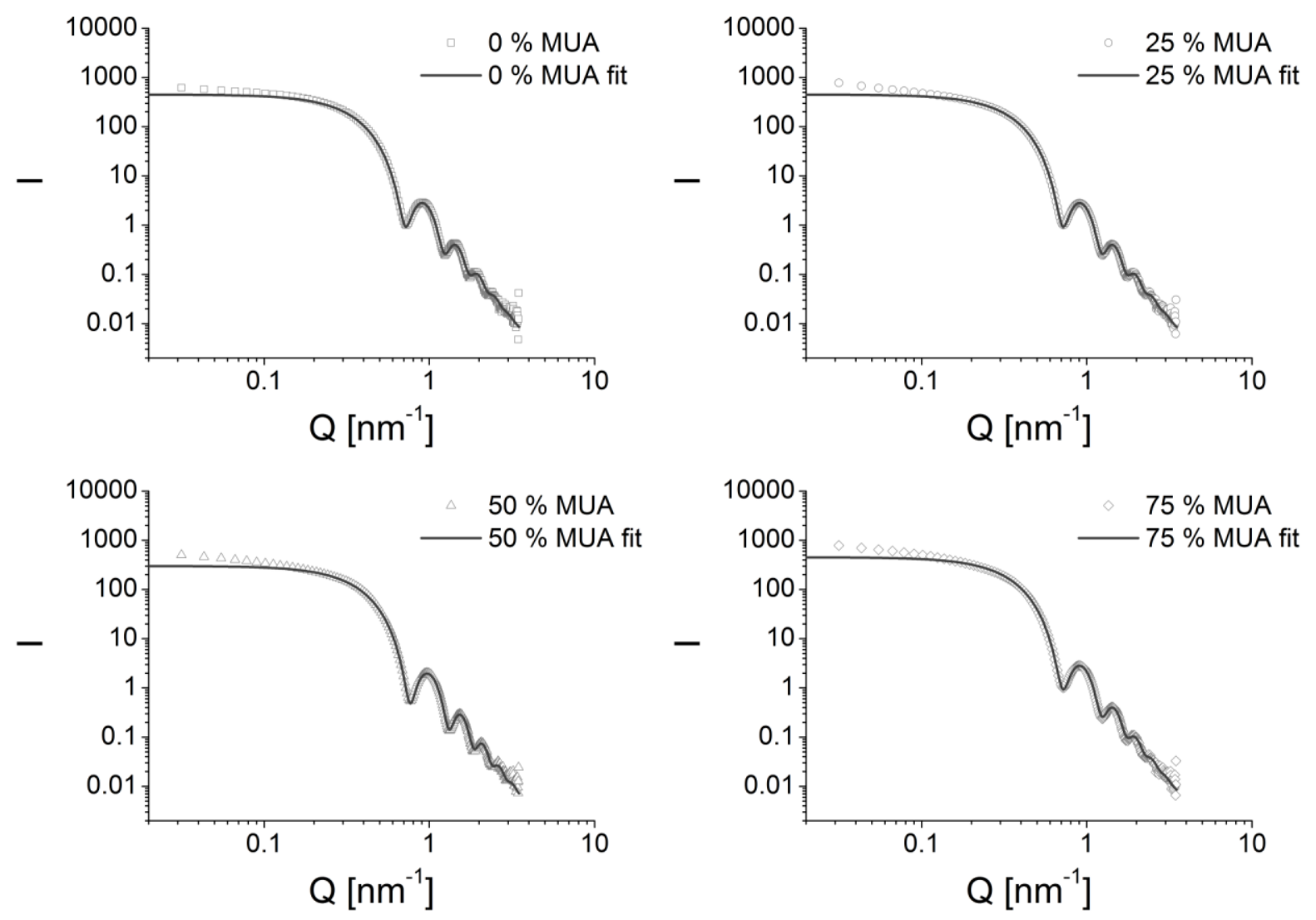

Figure 5. SAXS-Data of AuNP-conjugate dispersions at high concentrations $(c(\mathrm{AuNP})=200$ $\mathrm{nM}$ ) in water. Solid lines are fits to the data using the form factor of polydisperse spheres. Due to the low scattering contrast of the PEGMUA shell, only the Au core is visible. Aggregation would be visible by a strong increase of the scattering intensity at very low wave vector transfer Q.

The high colloidal stability of AuNP conjugates with PEGMUA/MUA mixtures is in stark contrast to that of AuNP coated with mixtures of a PEG-thiol of the same length but without $\mathrm{C}_{10^{-}}$ spacer ( $\alpha$-Methoxy- $\omega$-mercaptopoly(ethylene glycol), 2kDa, PEGSH) and MUA (Figure 6). After four centrifugations, all mixtures of PEGSH with MUA were aggregated irreversibly, visible by a broadening and redshift of the plasmon band and decrease of absorbance. 
Redispersion of the aggregated samples by ultrasonication was impossible. Only AuNP coated with $100 \%$ PEGSH, i.e. when no MUA was added, were stable. In contrast, all AuNP coated with PEGMUA/MUA mixtures showed no signs of aggregation and only AuNP coated with $100 \%$ MUA aggregated.
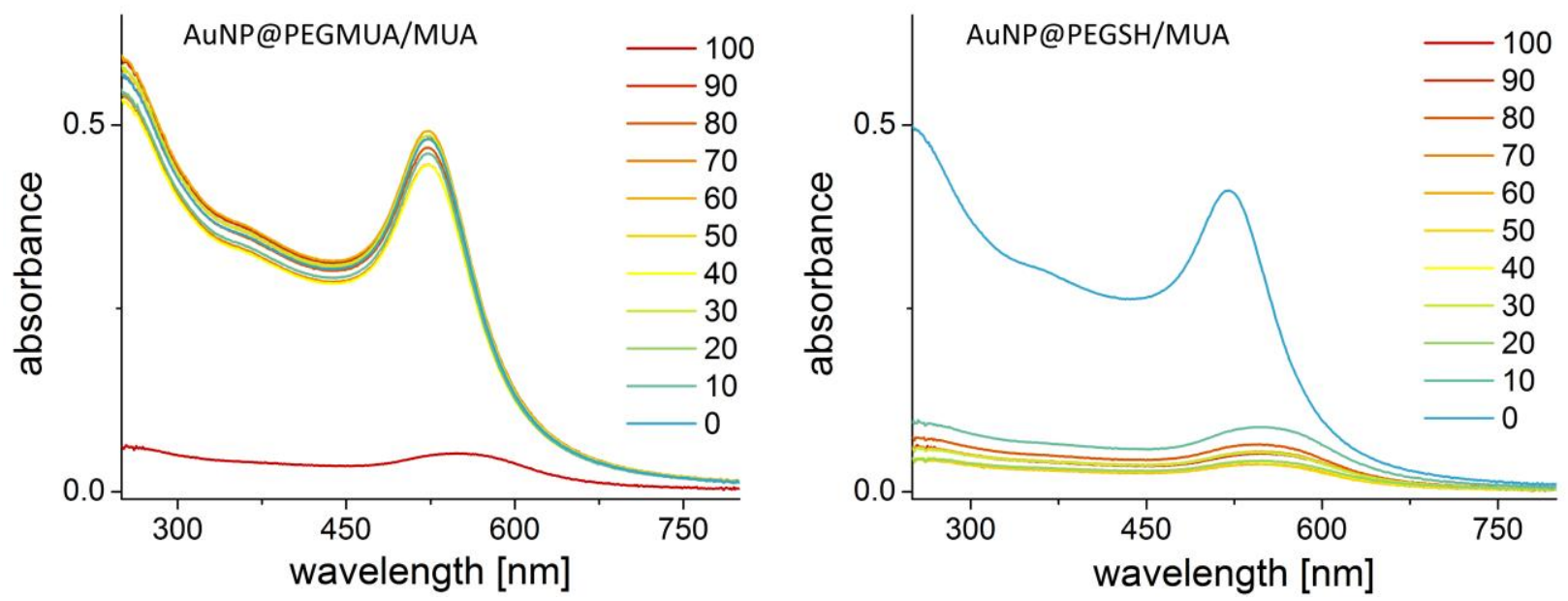

Figure 6. Absorbance spectra of AuNP conjugates after four centrifugations and redispersion (20,000 $\mathrm{g}$ x $20 \mathrm{~min}$ at room temperature). The initial AuNP concentrations were $\sim 5 \mathrm{nM}$, the final concentrations of stable samples were $\sim 150 \mathrm{nM}$. The concentrated samples were diluted for UV/Vis measurements. AuNP coated with PEGMUA/MUA mixtures (left) were stable and showed no signs of aggregation, whereas AuNP coated with mixtures of PEGSH and MUA were all aggregated irreversibly. The mole percent of MUA used in the synthesis of the conjugates are indicated by the color code.

These observations demonstrate the remarkable difference in competitive adsorption behavior of PEGMUA and PEGSH. Several recent studies have shown that PEGSH ligands can be competitively displaced by small alkyl thiols. Especially Smith et al. have recently provided a detailed analysis based on quantitative NMR that demonstrated the ability of MUA to competitively displace PEGSH-ligands. ${ }^{44}$ This displacement or competitive adsorption took 
place regardless of whether MUA was added simultaneously with the PEGSH ligand or to AuNP that were already functionalized with PEGSH. The observation that even mixtures with just $10 \%$ MUA and $90 \%$ PEGSH do not provide colloidal stability for AuNP confirms that the adsorption of MUA must be kinetically and thermodynamically strongly favored over PEGSH adsorption. Thus, a controlled synthesis of mixed ligand layers with MUA and PEGSH is impossible. In contrast, PEGMUA/MUA mixtures provide colloidal stability even with just $10 \%$ PEGMUA. Tailoring the molecular structure of the PEG by introducing a $\mathrm{C}_{10}$-spacer completely changes the adsorption behavior to a much more balanced situation.

To test the steric effect of the PEG-moiety on the colloidal stability, we compared AuNP functionalized with different ligands. AuNP stabilized with just MUA, or PEGMUA with $716 \mathrm{Da}$ (PEGMUA716) showed significant aggregation after centrifugation as indicated by DLS (Figure 7A). This resulted in significant concentration losses (85-90\% in the case of MUA, 35-40\% for PEGMUA716). In contrast, PEGMUA with 2000 Da or 5000 Da provided sufficient stabilization and no indications of aggregates were observed (Figure 7B, see also SAXS results in Figure 5 and Figure S2). The DLS experiments confirm the increasing thickness of the ligand shell using PEGMUA5k instead of PEGMUA2k and that PEGMUA716 is not providing sufficient steric stabilization to maintain colloidal stability during repeated centrifugation. The size distributions of AuNP@MUA and AuNP@PEGMUA716 are bimodal, indicating the presence of aggregates. They can therefore not be used to determine the thickness of the respective ligand shells. 

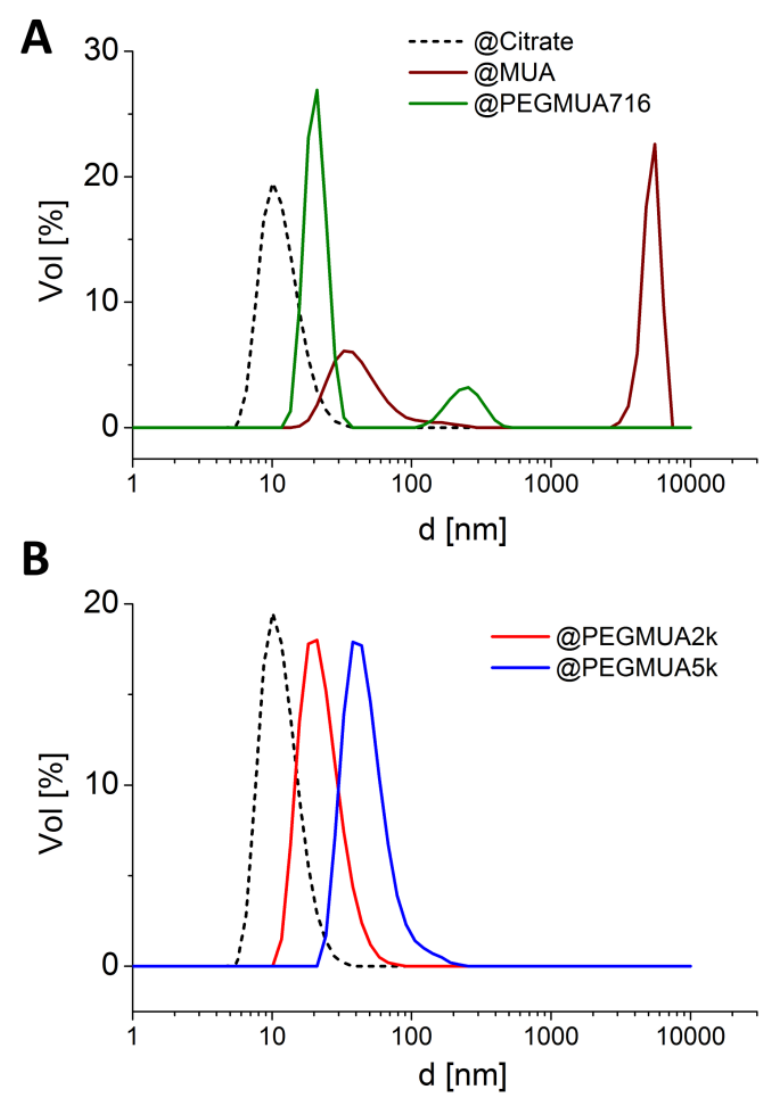

Figure 7. DLS measurements were performed to evaluate the mechanical stability of AuNP functionalized with different ligands as indicated by the color code. After 4 centrifugations (20,000 g x $20 \mathrm{~min}$ at room temperature) and redispersion the AuNP were analyzed by DLS. DLS results of AuNP@Citrate particles (dashed lines) that were not centrifuged are shown for comparison. The starting AuNP concentrations of all samples were $3 \mathrm{nM}$. After the centrifugations, that are used to purify and concentrate the samples, the AuNP concentrations of AuNP@PEGMUA2k and AuNP@PEGMUA5k were 16 nM, AuNP@PEGMUA716 were 10 nM and AuNP@MUA were 2 nM. Broad peaks and peaks at large hydrodynamic diameters in subfigure A indicate the presence of aggregates in the samples.

Thus, the incorporation of PEGMUA with a minimum molecular weight in the range of 2000 Da into the mixed ligand layers is necessary for an enhanced stabilization. MUA or short 
$\mathrm{EG}_{\mathrm{x}} \mathrm{MUAS}$ alone do not provide comparable stabilization of AuNP in this size range $(>10 \mathrm{~nm}$ diameter).

\section{Chemical stability}

Oxidative etching with cyanide was used to probe the accessibility of the AuNP's surface. The experiments were performed as described and discussed previously. ${ }^{14}$ In that study we demonstrated that the stability against oxidative etching dramatically increases with increasing length of a hydrophobic spacer. As a control experiment for our present study, we compared the stabilization provided by PEGMUA and by a commercial PEGSH ligand. As expected, the stabilization provided by PEGMUA is much higher: AuNP@PEGSH conjugates are etched completely within a few minutes, while only $\sim 20 \%$ of the particle population of AuNP@PEGMUA conjugates are etched, even after several hours (Figure 8). The pronounced stability of the AuNP@PEGMUA conjugates is most likely provided by the inner hydrophobic layer, which is formed at the AuNP surface by the alkylene spacers. Also, due to hydrophobic interactions between the spacers the PEGMUA ligands are probably more densely packed than PEGSH ligands (compare Figure 1) and, thus, provide additional stabilization. A high grafting density of PEGMUA $\left(\sim 3 \mathrm{~nm}^{-2}\right)$ in contrast to PEGSH was recently demonstrated with gold nanorods, strongly supporting this conclusion. ${ }^{64}$ 


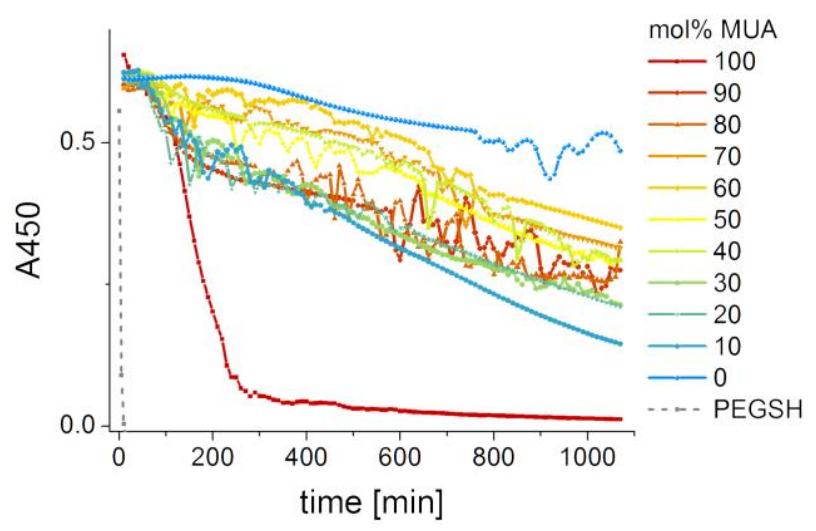

Figure 8. Stability of different AuNP conjugates in the presence of $200 \mathrm{mM} \mathrm{KCN}$. The absorbance at $450 \mathrm{~nm}, \mathrm{~A} 450$, reflects the concentration of the AuNP that decreases during the etching reaction. The assumed mole percent MUA in PEGMUA/MUA mixed conjugates are indicated by the color code. The data for AuNP@PEGSH in the presence of $100 \mathrm{mM}$ KCN are shown for comparison (grey squares, dashed line). AuNP coated with just MUA (100\% MUA) are not stable in the presence of high electrolyte concentrations and the decrease of their concentration is caused not only by etching but also by aggregation and sedimentation.

Comparing the oxidative etching of different mixed-layer MUA/PEGMUA-AuNP conjugates, a high stabilization was observed for all MUA ratios (Figure 8). This underlines that the PEGmoieties in the ligand layer are not decisive for the conjugate's stability against oxidative etching but the molecular structure at the particle's surface. Despite the decrease in PEG-density with increasing MUA ratio, the stability of the conjugates remains and no clear correlation of the MUA fraction and stability against etching was observed. On the other hand, the results suggest that the packing density of PEGMUA is not much lower than that of MUA, because otherwise the stability should increase with the MUA ratio. The same effects were observed for the competitive displacement with DTT (Figure S3), confirming these conclusions. AuNP coated with just MUA are not as stable as AuNP coated with PEGMUA/MUA mixtures because their 
stabilization is mainly based on electrostatic repulsion provided by the carboxylate groups. This electrostatic repulsion is screened at high electrolyte concentrations leading to aggregation of the AuNP, similar to the well-known electrolyte induced aggregation of citrate stabilized AuNP. This behavior was also observed in both experiments, stability against oxidative etching and stability against competitive displacement with DTT (tested in the presence of $0.4 \mathrm{M} \mathrm{NaCl}$ ), and the explanation is the same.

It can be concluded that optimal stabilization requires both, sufficient steric stabilization and a high grafting density of strongly bound ligands to form a hydrophobic inner shell. Coadsorption of sufficiently large PEG-polymers provides steric stabilization against strong mechanical forces and at high electrolyte concentrations. MUA adsorption provides chemical stabilization against competitive displacement and other unwanted reactions at the particles' surface by forming an inner hydrophobic layer with high density. In PEGMUA both effects are combined and we assume that the design principle can be generalized.

\section{Stability in serum and interactions with cells}

Because the use of mixed ligand layers is a popular strategy especially in nanomedicine, we tested the stability of MUA/PEGMUA-AuNP conjugates in serum and their interaction with mammalian cells. In human serum, no destabilization of the conjugates was observed (tested for 10 days), neither at $37^{\circ} \mathrm{C}$ nor at room temperature (Figure S4). The uptake of the conjugates by prostate cancer PCR3 cells was tested by incubation of the cells with the desired concentrations of AuNP conjugates. After $1 \mathrm{~h}$ and $24 \mathrm{~h}$ incubation, the cells were washed and their gold content determined by elemental analysis as described in the experimental section. AuNP uptake slightly increased with increasing MUA ratio, but was very low $(<1 \%$ of the gold added) for all samples (Figure 9). Similar results were obtained with A459 (lung cancer cells) and LNCaP (prostate 
cancer) cells (data not shown). The effect of AuNP concentration was tested for AuNP conjugates with $75 \%$ MUA because these showed the highest uptake. To determine their relative uptake, the gold content of the medium was also analyzed. The relative uptake (gold content in the cells divided by the total gold content in cells and medium) decreased with increasing AuNPconcentration, indicating low, unspecific uptake (Figure 9).
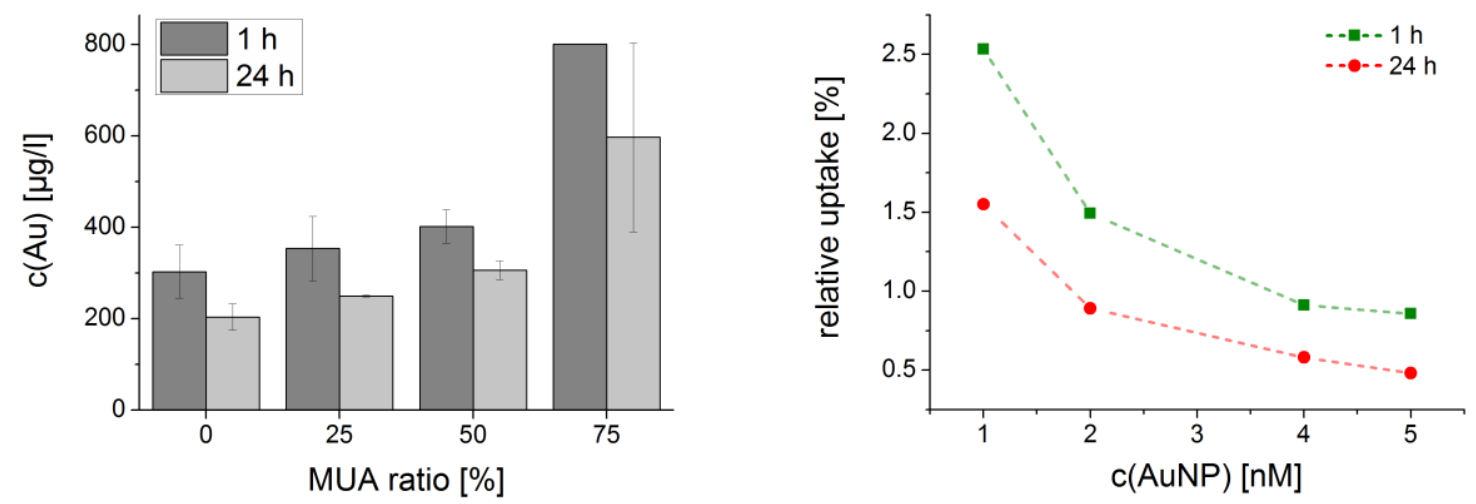

Figure 9. Left: Uptake of AuNP $(c(\mathrm{AuNP})=20 \mathrm{nM})$ with mixed ligand layers by PCR3 cells, 1 and $24 \mathrm{~h}$ after administration. The gold content was determined by elemental analysis as described in the experimental section. The assumed (based on the synthesis conditions) MUA mole percent in the mixed ligand layer of PEGMUA and MUA are indicated. Right: Relative uptake of AuNP@PEGMUA_MUA75 (assumed $75 \%$ MUA in the mixed ligand layer) for different AuNP concentrations and at different times after administration as indicated.

The uptake and cellular fate of AuNP is not completely understood, yet. ${ }^{65}$ PEG shells are known to reduce unspecific AuNP uptake and thus, coadsorption of MUA could be an interesting tool to tailor and study the surface chemistry of bioconjugates. In our cell uptake experiments we found no signs of stress or toxicity (e.g. no changed morphology of the cells was observed with optical microscopy), even at high AuNP concentrations (tested up to $20 \mathrm{nM}$ in the cell medium). Toxicity tests confirmed these findings (Figure S5). Colloids with stabilities that enable very 
high concentrations without inducing any toxicity are highly desirable for various applications in nanomedicine (e.g. CT-imaging and plasmonic photothermal therapy, PPTT) ${ }^{66-68}$ On the other hand, high stability is often compromised with increasing complexity of the system, e.g. by binding of functional molecules (peptides, DNA, antibodies, etc.) or labels. Finding the optimum balance of functionality and stability is usually a challenging task. The design proposed in this study represents a highly stable nanoparticle platform. Our experiments strongly indicate that the stability of bioconjugates with mixed ligand layers in general can be improved significantly by using PEGs and other ligands with hydrophobic alkylene spacers. These spacers (e.g. in form of MUA) can be coupled easily to PEG ${ }^{14,51}$ and biomolecules (PEGs, peptides and oligonucleotides are also commercially available with undecanyl-spacers, at least as custom synthesis). MUA in SAMs can be activated for EDC coupling and other coupling strategies, ${ }^{69}$ possibly allowing for direct coupling strategies with AuNP@PEGMUA/MUA conjugates. Nieves et al. recently demonstrated that the incorporation of SH-MUAEG5-N3 into mixed ligand layers on AuNP enables the versatile functionalization, e.g. by introducing a maleimide group via click chemistry. ${ }^{70}$ Such a ligand is also expected not to compromise the high stabilization of the mixed layers underlining the flexibility of the approach.

We have shown that functional peptides can be incorporated into mixed ligand layers with PEGMUA. ${ }^{71}$ Importantly, the lower number of conjugated peptides (because of the coadsorption of PEGMUA) did not result in decreased functionality. In contrast, both, the stability and functionality of the conjugates were improved with increasing PEGMUA ratio. The peptides in that previous study were not bound via a hydrophobic spacer and we believe that by using such a spacer the control in the synthesis and stability of these mixed conjugates could be even further improved. In general, short functional peptides should be good candidates for the synthesis of 
functional mixed ligand layers as they typically have a similar size as PEGMUA with $2 \mathrm{kDa}$ or 5 $\mathrm{kDa}$ and via additional spacers (e.g. as oligoglycines) their presentation at the ligand shell surface can be tuned.

\section{CONCLUSION}

We have shown that by using a hydrophobic alkylene spacer comprising 10 methylene units $\left(\mathrm{C}_{10}\right)$, AuNP with tunable mixed ligand layers can be synthesized. The surface charge of these conjugates can be tuned without impairing their high stability against competitive displacement, oxidative etching, centrifugation, and against electrolyte concentration and protein adsorption in serum. In consequence, highly concentrated samples of these conjugates can be prepared with immaculate colloidal integrity, i.e. free of aggregates. Importantly, our study underlines that comparable stability of mixed ligand layer conjugates cannot be achieved by using PEG-thiol ligands without a hydrophobic spacer. Additional experiments demonstrated that the PEG part of the ligand layer is responsible for the colloidal stability, whereas the inner hydrophobic layer consisting of alkylene chains provides chemical stability. These findings are especially relevant for the synthesis of nanoconjugates for applications in medicine and bionanotechnology, where the use of mixed ligand layers including PEG-thiols as stabilizers is a popular strategy.

\section{ASSOCIATED CONTENT}

TEM characterization of all AuNP batches, additional SAXS results, UV/Vis monitoring of competitive ligand displacement with dithiothreitol, UV/Vis spectra of AuNP conjugates in serum after different incubation times, results of lactate dehydrogenase (LDH) activity assay 
with different AuNP conjugates. This material is available free of charge via the Internet at http://pubs.acs.org.

\section{AUTHOR INFORMATION}

\section{Corresponding Author}

*schulzf@chemie.uni-hamburg.de

\section{Present Addresses}

\# European Molecular Biology Laboratory (EMBL) c/o DESY

Notkestr. 85, Geb. 25a

22607 Hamburg

Germany

\section{Author Contributions}

The manuscript was written through contributions of all authors. All authors have given approval to the final version of the manuscript.

\section{Notes}

The authors declare no competing financial interest.

\section{ACKNOWLEDGMENTS}

We acknowledge financial support from the German Research Foundation (DFG) via the Cluster of Excellence "Centre for Ultrafast Imaging" (CUI). F.S. is supported by the DFG via the project SCHU 3019/2-1. The authors thank Frank Meyberg and his team for GF-AAS measurements, and Elif Metin, Marcus von der Au and Andrea Pietsch for assistance with 
preliminary experimental work. We thank Johannes Möller and Andrew J. Smith for excellent support for the SAXS experiments. We acknowledge Diamond Light Source for time on I22 under proposal SM11192.

\section{REFERENCES}

(1) Boles, M. A.; Ling, D.; Hyeon, T.; Talapin, D. V. The surface science of nanocrystals. Nat. Mater. 2016, 15, 141-153.

(2) Jokerst, J. V.; Lobovkina, T.; Zare, R. N.; Gambhir, S. S. Nanoparticle PEGylation for imaging and therapy. Nanomedicine 2011, 6, 715-728.

(3) Love, J. C.; Estroff, L. A.; Kriebel, J. K.; Nuzzo, R. G.; Whitesides, G. M. SelfAssembled Monolayers of Thiolates on Metals as a Form of Nanotechnology. Chem. Rev. 2005, 105, 1103-1170.

(4) Moyano, D. F.; Rotello, V. M. Nano Meets Biology: Structure and Function at the Nanoparticle Interface. Langmuir 2011, 27, 10376-10385.

(5) Nel, A. E.; Madler, L.; Velegol, D.; Xia, T.; Hoek, E. M. V.; Somasundaran, P.; Klaessig, F.; Castranova, V.; Thompson, M. Understanding biophysicochemical interactions at the nano-bio interface. Nat. Mater. 2009, 8, 543-557.

(6) Sperling, R. A.; Parak, W. J. Surface modification, functionalization and bioconjugation of colloidal inorganic nanoparticles. Phil. Trans. R. Soc. A 2010, 368, 1333-1383.

(7) Zhang, W. Nanoparticle Aggregation: Principles and Modeling. Nanomaterial: Impacts on Cell Biology and Medicine, 2014, 19-43.

(8) Aberasturi, D. J. de; Serrano-Montes, A. B.; Liz-Marzán, L. M. Modern Applications of Plasmonic Nanoparticles: From Energy to Health. Adv. Opt. Mater. 2015, 3, 602-617.

(9) Daniel, M.-C.; Astruc, D. Gold Nanoparticles: Assembly, Supramolecular Chemistry, Quantum-Size-Related Properties, and Applications toward Biology, Catalysis, and Nanotechnology. Chem. Rev. 2004, 104, 293-346.

(10) Dreaden, E. C.; Alkilany, A. M.; Huang, X.; Murphy, C. J.; El-Sayed, M. A. The golden age: gold nanoparticles for biomedicine. Chem. Soc. Rev. 2012, 41, 2740-2779.

(11) Mout, R.; Moyano, D. F.; Rana, S.; Rotello, V. M. Surface functionalization of nanoparticles for nanomedicine. Chem. Soc. Rev. 2012, 41, 2539-2544.

(12) Ghosh, S. K.; Pal, T. Interparticle Coupling Effect on the Surface Plasmon Resonance of Gold Nanoparticles: From Theory to Applications. Chem. Rev. 2007, 107, 4797-4862.

(13) Mei, B. C.; Oh, E.; Susumu, K.; Farrell, D.; Mountziaris, T. J.; Mattoussi, H. Effects of Ligand Coordination Number and Surface Curvature on the Stability of Gold Nanoparticles in Aqueous Solutions. Langmuir 2009, 25, 10604-10611.

(14) Schulz, F.; Vossmeyer, T.; Bastús, N. G.; Weller, H. Effect of the Spacer Structure on the Stability of Gold Nanoparticles Functionalized with Monodentate Thiolated Poly(ethylene glycol) Ligands. Langmuir 2013, 29, 9897-9908.

(15) Dobrovolskaia, M. A.; Patri, A. K.; Zheng, J.; Clogston, J. D.; Ayub, N.; Aggarwal, P.; Neun, B. W.; Hall, J. B.; McNeil, S. E. Interaction of colloidal gold nanoparticles with human blood: effects on particle size and analysis of plasma protein binding profiles. Nanomedicine 2009, 5, 106-117. 
(16) Mahmoudi, M.; Lynch, I.; Ejtehadi, M. R.; Monopoli, M. P.; Bombelli, F. B.; Laurent, S. Protein-Nanoparticle Interactions: Opportunities and Challenges. Chem. Rev. 2011, 111, 5610-5637.

(17) Natte, K.; Friedrich, J. F.; Wohlrab, S.; Lutzki, J.; Klitzing, R. von; Österle, W.; Orts-Gil, G. Impact of polymer shell on the formation and time evolution of nanoparticle-protein corona. Colloids Surf., B 2013, 104, 213-220.

(18) Bishop, K. J. M.; Wilmer, C. E.; Soh, S.; Grzybowski, B. A. Nanoscale Forces and Their Uses in Self-Assembly. Small 2009, 5, 1600-1630.

(19) Duchesne, L.; Gentili, D.; Comes-Franchini, M.; Fernig, D. G. Robust Ligand Shells for Biological Applications of Gold Nanoparticles. Langmuir 2008, 24, 13572-13580.

(20) Karakoti, A. S.; Das, S.; Thevuthasan, S.; Seal, S. PEGylated Inorganic Nanoparticles. Angew. Chem. Int. Ed. 2011, 50, 1980-1994.

(21) Liu, Y.; Shipton, M. K.; Ryan, J.; Kaufman, E. D.; Franzen, S.; Feldheim, D. L. Synthesis, Stability, and Cellular Internalization of Gold Nanoparticles Containing Mixed Peptide-Poly(ethylene glycol) Monolayers. Anal. Chem. 2007, 79, 2221-2229.

(22) Maus, L.; Spatz, J. P.; Fiammengo, R. Quantification and Reactivity of Functional Groups in the Ligand Shell of PEGylated Gold Nanoparticles via a Fluorescence-Based Assay. Langmuir 2009, 25, 7910-7917.

(23) Niidome, T.; Yamagata, M.; Okamoto, Y.; Akiyama, Y.; Takahashi, H.; Kawano, T.; Katayama, Y.; Niidome, Y. PEG-modified gold nanorods with a stealth character for in vivo applications. J. Control. Release 2006, 114, 343-347.

(24) Otsuka, H.; Nagasaki, Y.; Kataoka, K. PEGylated nanoparticles for biological and pharmaceutical applications. Adv. Drug Deliv. Rev. 2003, 55, 403-419.

(25) Pengo, P.; Baltzer, L.; Pasquato, L.; Scrimin, P. Substrate Modulation of the Activity of an Artificial Nanoesterase Made of Peptide-Functionalized Gold Nanoparticles. Angew. Chem. Int. Ed. 2007, 46, 400-404.

(26) Sapsford, K. E.; Algar, W. R.; Berti, L.; Gemmill, K. B.; Casey, B. J.; Oh, E.; Stewart, M. H.; Medintz, I. L. Functionalizing Nanoparticles with Biological Molecules: Developing Chemistries that Facilitate Nanotechnology. Chem. Rev. 2013, 113, 1904-2074.

(27) Na, H. B.; Palui, G.; Rosenberg, J. T.; Ji, X.; Grant, S. C.; Mattoussi, H. Multidentate Catechol-Based Polyethylene Glycol Oligomers Provide Enhanced Stability and Biocompatibility to Iron Oxide Nanoparticles. ACS Nano 2012, 6, 389-399.

(28) Palui, G.; Na, H. B.; Mattoussi, H. Poly(ethylene glycol)-Based Multidentate Oligomers for Biocompatible Semiconductor and Gold Nanocrystals. Langmuir 2012, 28, 27612772.

(29) Stewart, M. H.; Susumu, K.; Mei, B. C.; Medintz, I. L.; Delehanty, J. B.; Blanco-Canosa, J. B.; Dawson, P. E.; Mattoussi, H. Multidentate Poly(ethylene glycol) Ligands Provide Colloidal Stability to Semiconductor and Metallic Nanocrystals in Extreme Conditions. $J$. Am. Chem. Soc. 2010, 132, 9804-9813.

(30) Thierry, B.; Griesser, H. J. Dense PEG layers for efficient immunotargeting of nanoparticles to cancer cells. J. Mater. Chem. 2012, 22, 8810-8819.

(31) Uyeda, H. T.; Medintz, I. L.; Jaiswal, J. K.; Simon, S. M.; Mattoussi, H. Synthesis of Compact Multidentate Ligands to Prepare Stable Hydrophilic Quantum Dot Fluorophores. J. Am. Chem. Soc. 2005, 127, 3870-3878. 
(32) Chen, X.; Qoutah, W. W.; Free, P.; Hobley, J.; Fernig, D. G.; Paramelle, D. Features of Thiolated Ligands Promoting Resistance to Ligand Exchange in Self-Assembled Monolayers on Gold Nanoparticles. Aust. J. Chem. 2012, 65, 266-274.

(33) Lévy, R. Peptide-Capped Gold Nanoparticles: Towards Artificial Proteins. ChemBioChem 2006, 7, 1141-1145.

(34) Lévy, R.; Thanh, N. T. K.; Doty, R. C.; Hussain, I.; Nichols, R. J.; Schiffrin, D. J.; Brust, M.; Fernig, D. G. Rational and Combinatorial Design of Peptide Capping Ligands for Gold Nanoparticles. J. Am. Chem. Soc. 2004, 126, 10076-10084.

(35) Rouhana, L. L.; Jaber, J. A.; Schlenoff, J. B. Aggregation-Resistant Water-Soluble Gold Nanoparticles. Langmuir 2007, 23, 12799-12801.

(36) Susumu, K.; Oh, E.; Delehanty, J. B.; Blanco-Canosa, J. B.; Johnson, B. J.; Jain, V.; William Judson Hervey, I.; Algar, W. R.; Boeneman, K.; Dawson, P. E.; et al. Multifunctional Compact Zwitterionic Ligands for Preparing Robust Biocompatible Semiconductor Quantum Dots and Gold Nanoparticles. J. Am. Chem. Soc. 2011, 133, 9480-9496.

(37) Liu, X.; Huang, H.; Jin, Q.; Ji, J. Mixed Charged Zwitterionic Self-Assembled Monolayers as a Facile Way to Stabilize Large Gold Nanoparticles. Langmuir 2011, 27, 5242-5251.

(38) Liu, X.; Li, H.; Jin, Q.; Ji, J. Surface Tailoring of Nanoparticles via Mixed-Charge Monolayers and Their Biomedical Applications. Small 2014, 10, 4230-4242.

(39) Stewart, A.; Zheng, S.; McCourt, M. R.; Bell, S. E. J. Controlling Assembly of Mixed Thiol Monolayers on Silver Nanoparticles to Tune Their Surface Properties. ACS Nano 2012, 6, 3718-3726.

(40) Van Lehn, R. C.; Alexander-Katz, A. Structure of Mixed-Monolayer-Protected Nanoparticles in Aqueous Salt Solution from Atomistic Molecular Dynamics Simulations. J. Phys. Chem. C 2013, 117, 20104-20115.

(41) Zaccari, I.; Catchpole, B. G.; Laurenson, S. X.; Davies, A. G.; Wälti, C. Improving the Dielectric Properties of Ethylene-Glycol Alkanethiol Self-Assembled Monolayers. Langmuir 2014, 30, 1321-1326.

(42) Larson-Smith, K.; Pozzo, D. C. Competitive Adsorption of Thiolated Poly(ethylene glycol) and Alkane-Thiols on Gold Nanoparticles and Its Effect on Cluster Formation. Langmuir 2012, 28, 13157-13165.

(43) Siriwardana, K.; Gadogbe, M.; Ansar, S. M.; Vasquez, E. S.; Collier, W. E.; Zou, S.; Walters, K. B.; Zhang, D. Ligand Adsorption and Exchange on Pegylated Gold Nanoparticles. J. Phys. Chem. C 2014, 118, 11111-11119.

(44) Smith, A. M.; Marbella, L. E.; Johnston, K. A.; Hartmann, M. J.; Crawford, S. E.; Kozycz, L. M.; Seferos, D. S.; Millstone, J. E. Quantitative Analysis of Thiolated Ligand Exchange on Gold Nanoparticles Monitored by 1H NMR Spectroscopy. Anal. Chem. 2015, 87, 2771-2778.

(45) Rahme, K.; Chen, L.; Hobbs, R. G.; Morris, M. A.; O’Driscoll, C.; Holmes, J. D. PEGylated gold nanoparticles: polymer quantification as a function of PEG lengths and nanoparticle dimensions. R. Soc. Chem. Adv. 2013, 3, 6085-6094.

(46) Xia, X.; Yang, M.; Wang, Y.; Zheng, Y.; Li, Q.; Chen, J.; Xia, Y. Quantifying the Coverage Density of Poly(ethylene glycol) Chains on the Surface of Gold Nanostructures. ACS Nano 2012, 6, 512-522. 
(47) Agasti, S. S.; You, C.-C.; Arumugam, P.; Rotello, V. M. Structural control of the monolayer stability of water-soluble gold nanoparticles. J. Mater. Chem. 2008, 18, 70-73.

(48) Tsai, D.-H.; Shelton, M. P.; DelRio, F. W.; Elzey, S.; Guha, S.; Zachariah, M. R.; Hackley, V. A. Quantifying dithiothreitol displacement of functional ligands from gold nanoparticles. Anal. Bioanal. Chem. 2012, 404, 3015-3023.

(49) Tsai, D.-H.; Davila-Morris, M.; DelRio, F. W.; Guha, S.; Zachariah, M. R.; Hackley, V. A. Quantitative Determination of Competitive Molecular Adsorption on Gold Nanoparticles Using Attenuated Total Reflectance-Fourier Transform Infrared Spectroscopy. Langmuir 2011, 27, 9302-9313.

(50) Tsai, D.-H.; DelRio, F. W.; MacCuspie, R. I.; Cho, T. J.; Zachariah, M. R.; Hackley, V. A. Competitive Adsorption of Thiolated Polyethylene Glycol and Mercaptopropionic Acid on Gold Nanoparticles Measured by Physical Characterization Methods. Langmuir 2010, 26, 10325-10333.

(51) Larson, T. A.; Joshi, P. P.; Sokolov, K. Preventing Protein Adsorption and Macrophage Uptake of Gold Nanoparticles via a Hydrophobic Shield. ACS Nano 2012, 6, 9182-9190.

(52) Ghosh, P.; Han, G.; De, M.; Kim, C. K.; Rotello, V. M. Gold nanoparticles in delivery applications. Adv. Drug Delivery Rev. 2008, 60, 1307-1315.

(53) Kanaras, A. G.; Kamounah, F. S.; Schaumburg, K.; Kiely, C. J.; Brust, M. Thioalkylated tetraethylene glycol: a new ligand for water soluble monolayer protected gold clusters. Chem. Commun. 2002, 2294-2295.

(54) Simpson, C. A.; Agrawal, A. C.; Balinski, A.; Harkness, K. M.; Cliffel, D. E. Short-Chain PEG Mixed Monolayer Protected Gold Clusters Increase Clearance and Red Blood Cell Counts. ACS Nano 2011, 5, 3577-3584.

(55) Schulz, F.; Homolka, T.; Bastús, N. G.; Puntes, V.; Weller, H.; Vossmeyer, T. Little Adjustments Significantly Improve the Turkevich Synthesis of Gold Nanoparticles. Langmuir 2014, 30, 10779-10784.

(56) Haiss, W.; Thanh, N. T. K.; Aveyard, J.; Fernig, D. G. Determination of Size and Concentration of Gold Nanoparticles from UV-Vis Spectra. Anal. Chem. 2007, 79, 42154221.

(57) Neutron, X-rays and Light. Scattering Methods Applied to Soft Condensed Matter; Lindner, P.; Zemb, T., Eds.; 1st ed.; North Holland, 2001.

(58) Harder, P.; Grunze, M.; Dahint, R.; Whitesides, G. M.; Laibinis, P. E. Molecular Conformation in Oligo(ethylene glycol)-Terminated Self-Assembled Monolayers on Gold and Silver Surfaces Determines Their Ability To Resist Protein Adsorption. J. Phys. Chem. B 1998, 102, 426-436.

(59) Comenge, J.; Puntes, V. The role of PEG conformation in mixed layers: from protein corona substrate to steric stabilization avoiding protein adsorption. Science Open Research 2015, 1-10.

(60) Bain, C. D.; Whitesides, G. M. Formation of monolayers by the coadsorption of thiols on gold: variation in the length of the alkyl chain. J. Am. Chem. Soc. 1989, 111, 7164-7175.

(61) Kakiuchi, T.; Iida, M.; Gon, N.; Hobara, D.; Imabayashi, S.; Niki, K. Miscibility of Adsorbed 1-Undecanethiol and 11-Mercaptoundecanoic Acid Species in Binary SelfAssembled Monolayers on Au(111). Langmuir 2001, 17, 1599-1603.

(62) Lavrich, D. J.; Wetterer, S. M.; Bernasek, S. L.; Scoles, G. Physisorption and Chemisorption of Alkanethiols and Alkyl Sulfides on Au(111). J. Phys. Chem. B 1998, $102,3456-3465$. 
(63) Hostetler, M. J.; Wingate, J. E.; Zhong, C.-J.; Harris, J. E.; Vachet, R. W.; Clark, M. R.; Londono, J. D.; Green, S. J.; Stokes, J. J.; Wignall, G. D.; et al. Alkanethiolate Gold Cluster Molecules with Core Diameters from 1.5 to $5.2 \mathrm{~nm}$ : Core and Monolayer Properties as a Function of Core Size. Langmuir 1998, 14, 17-30.

(64) Schulz, F.; Friedrich, W.; Hoppe, K.; Vossmeyer, T.; Weller, H.; Lange, H. Effective PEGylation of gold nanorods. Nanoscale 2016, 8, 7296-7308.

(65) Lévy, R.; Shaheen, U.; Cesbron, Y.; Sée, V. Gold nanoparticles delivery in mammalian live cells: a critical review. Nano Rev. 2010, 1, 4889.

(66) Dykman, L.; Khlebtsov, N. Gold nanoparticles in biomedical applications: recent advances and perspectives. Chem. Soc. Rev. 2012, 41, 2256-2282.

(67) Huang, X.; Jain, P. K.; El-Sayed, I. H.; El-Sayed, M. A. Plasmonic photothermal therapy (PPTT) using gold nanoparticles. Laser Med. Sci. 2008, 23, 217-228.

(68) Lee, N.; Choi, S. H.; Hyeon, T. Nano-Sized CT Contrast Agents. Adv. Mater. 2013, 25, 2641-2660.

(69) Frey, B. L.; Robert M. Corn, and. Covalent Attachment and Derivatization of Poly(1lysine) Monolayers on Gold Surfaces As Characterized by Polarization-Modulation FTIR Spectroscopy. Anal. Chem. 1996, 68, 3187-3193.

(70) Nieves, D. J.; Azmi, N. S.; Xu, R.; Levy, R.; Yates, E. A.; Fernig, D. G. Monovalent maleimide functionalization of gold nanoparticles via copper-free click chemistry. Chem. Commun. 2014, 50, 13157-13160.

(71) Schulz, F.; Lutz, D.; Rusche, N.; Bastus, N. G.; Stieben, M.; Holtig, M.; Gruner, F.; Weller, H.; Schachner, M.; Vossmeyer, T.; et al. Gold nanoparticles functionalized with a fragment of the neural cell adhesion molecule L1 stimulate L1-mediated functions. Nanoscale 2013, 5, 10605-10617.

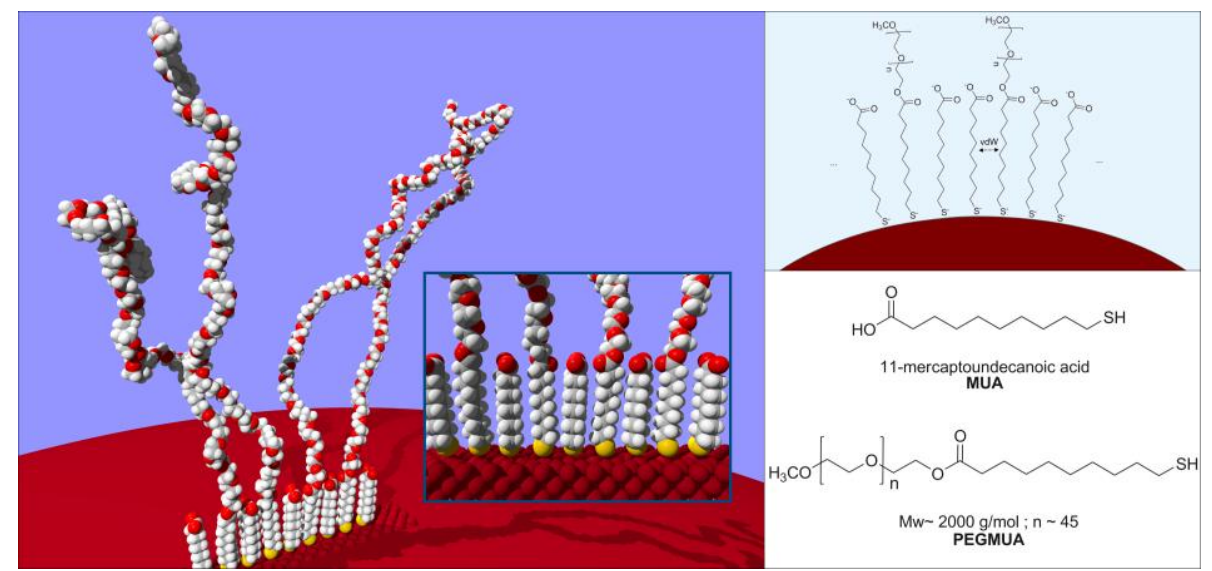

